\title{
Research on the Application of Painting Elements on Modern Graphic Design
}

\author{
Yun Qin \\ Yunnan Energy Vocational and Technical College, Qujing, Yunnan, 655001
}

Keywords: painting elements; modern graphic design; application study

\begin{abstract}
From the perspective of modern disciplines, painting art and graphic advertising design are two different disciplines of art, but also different natures of art, but fundamentally, the two are interrelated and mutually learnable. A good advertising design must have a good foundation of painting, be able to understand and be good at using painting elements. The visual effects of painting elements in advertising design reflect the different influences of different cultures.
\end{abstract}

\section{Introduction}

Modern print advertising design is developing very fast in our current social life. In the past, when we talked about the globalization and internationalization of advertising, it has always been considered a very fresh topic, but now it has become the most common topic but its The fashion trend has not been reduced. This popular design with traditional culture and traditional painting elements in modern print ads has gradually formed its own unique style. Both traditional Chinese painting and print advertising design exist as a kind of visual art. When showing the meaning of the times, Chinese traditional painting elements can be used as a kind of parent of Chinese graphic advertising creative performance. Starting from the aesthetic elements and creative thinking of traditional painting, we can discover the similarities between painting and print advertising. If the two can be combined in form and connotation, they can continue the lifeline of painting elements on the one hand, and make the Chinese print advertising design form a unique right to speak in international advertising. We have made relevant academic research on a series of problems in the understanding of painting elements and the application in modern print advertising design, so that it can be of great significance to the development of our modern print advertising design. The combination of the artistry of painting elements and the functionality of print advertising design contributes to the promotion of corporate brands and the formation of the designer's personal style. Therefore, in the process of learning, we explore the application of traditional Chinese painting elements in modern print advertising design, which allows us to understand that its significance in real life is very important and can not be ignored, and also helps us to further understand modern print advertising design. The future development prospects also have profound significance.

\section{Definition of Painting Element}

Painting refers to the use of brushes, paints and other tools on the walls, paper and various textiles to express the visual image and visual content that the author wants to convey through various forms of expression. This form of expression is diverse and rich. There is a different definition of the term element in all walks of life, and it also has great differences. Taking painting as an example, in the field of painting, an element refers to all things that constitute a visual composition. In simple terms, an element refers to a certain color or a kind of brush in a painting, and painting uses these elements to form a visual map that the author wants to express using various composition rules. In extension, an element can refer to all the tools in a painting, that is, materials, paintings, or the language of painting. Therefore, to a certain extent, the elements of painting can be extended and extended to any field, especially in modern graphic design, which can be arbitrarily converted into all elements of modern graphic design, such as design, technique, style and so on. With the rapid development of computer technology, the connection between painting and print advertising design is getting stronger and stronger. Even though there are various differences 
between the two, it has to be acknowledged that they are inextricably linked. . Wang Youjiang once said in a book that painting art provides various forms of elemental foundation for modern graphic design. However, with the progress and development of society, painting and graphic advertising design have gradually formed two different disciplines. Constantly strengthening the study of painting elements will contribute to the improvement and improvement of graphic design capabilities. Therefore, the design of graphic print advertising has a very important connection. If a person does not know how to appreciate the art of painting and does not know how to use painting elements, then he will not make any achievements in the field of modern graphic advertising design. Thirdly, in the early stage of graphic advertising design, it is also inseparable from the reference of painting elements. Before the graphic design, it is necessary to use the painting method to record the inspiration of the author in time to provide reference and help for graphic design. Finally, a successful print ad can be expressed through the art of painting to show the author's thoughts.

\section{Re-picking and Combining Painting Elements}

The combination and disassembly is the deconstruction. The theoretical basis of deconstruction is the lattice structure theory, which refers to a highly organized whole body formed by the object in the observer's mind. Taking the elements of ancient and modern paintings as objects, according to the needs of design, the decomposition and reconstruction of symbolic meanings, that is, the elements into mechanisms, schemas, symbols, etc., into the symbol storage, and then the design reconstruction, this process Deconstruction, for modern print advertising design, deconstruction is a very powerful design method. Of course, the structure is not arbitrary, but has the high-level characteristics that attach importance to internal structural factors and overall considerations. It breaks down the formal principles and forms of the orthodox modern design, and takes up the future design space with a new look. The immortal work of the world - IBM posters should be a must for print advertising designers. In this advertisement design by Paul Rand, we can see that she deconstructed the "Montage (" and "Collage") of the European modernist Picasso art pattern or aesthetic concept and Matisse's color system. The technique is applied to his design. As a "new advertiser" and "functionalist", Paul Rand made a clear boundary between "the design of content" and "the abstract design without content". His print advertisements usually lead the audience to actively explore and interpret their advertisements through the deconstruction of various painting elements, and feel the content conveyed by the works. Taking IBM's poster (Eye-Bee-M) as an example, Paul Rand is not simply using the painting elements directly to design posters, but to decompose and reconstruct the elements in these paintings by symbolic meaning. Give the graphic advertising design a personalized and stylized artistic charm. It is both typical and harmonious. It can show the context of design and the value of creation. It not only conforms to the development rules of science and art, but also meets the mind and acceptance of the audience.

\section{The Application of Painting Expression Language in Modern Print Advertising Design}

All the senses of touch, vision and needs, emotional thinking, etc. that the human body feels are all created by virtue. From the aspect of artistic creation, we can not only explore from the external form of the object, otherwise we can only grasp the object. The surface loses its essence. Gu Yuzhi, a famous painter from the Eastern Jin Dynasty, once said, "Where people are born and killed, there are those who are blinded by their eyes and those who have died before. It's true that they are shaped by God, The ink paintings also show the beauty of the human body, the beauty of the halo and the stalwart of the mountains and rocks. The contrast between the water and the static movement of the moon is reflected, creating a powerful visual impact. Therefore, for the design of print advertising, it is necessary to continuously combine the impact characteristics of painting, attract people's attention, attract attention, and raise awareness of people, so as to continuously gain understanding and recognition for the purpose of propaganda. .

In the book Art and Visual Perception, it has been argued that "a harmonious and unified organization group is formed by constant combination of various simple and complex elements, so 
the human mind is only in this social and physical environment. Will form an optimal state" [1]. In Chinese painting, the reason why the stone is angry and agile is because of the promotion of nature. The reason why the river pebble is beautifully shaped is because of the shaping of the river. The reason why the mountain is mighty is that there is a movement of the earth's crust... the static of the nature in the earth Beauty is formed by the various connections of nature, and they are not isolated. Compared with Western paintings, traditional Chinese ink paintings have the characteristics of rich emotions and long-distance artistic conception. In addition to this, there is a high degree of generalization ability, which leads to people's taste. Similarly, Chen Zhengda, a famous Chinese graphic designer, believes that painting can't express objects, and can only convey its artistic conception and connotation to readers through the power of pen and ink. Taking a reluctant work as an example, his works exemplify his Lao Zhuang thoughts, and he has the characteristics of innovation, learning and use. It is precisely because of this that his works can be easily read by readers all over the world. Understood. Like $\mathrm{Gu}$ Yuzhi, his works are a clever combination of "writing God" and "writing God", and the central idea of his works is cleverly expressed in objects. On the whole, traditional Chinese painting attaches great importance to the combination of form and spirit, and uses various combinations of objects to express the temperament, imposing and implication of beauty in the works. In the modern print advertising design, the use and reference of traditional Chinese painting elements are deepening, mainly in the use and application of the spirit and meaning.

With the continuous development of oil painting, the texture of oil painting has gradually evolved from a simple pigment filling to a performance tool for painting, and has become a reflection of the spirit of painting. The vision and touch of materials are an integral part of oil painting. First, texture and materials can express the essence of the work through visual problems. Once again, the human body can better sense the properties of the material through the touch, including the softness and hardness of the material, the degree of dryness and humidity, and the various feelings brought by the texture. Finally, materials and textures can give the body different psychological experiences, such as whether an object has vitality? Is it chilly? Is it soft? Therefore, in the design of modern print advertising, we must pay more attention to the influence of materials and texture on the design effect, and learn to use the unique advantages of materials and texture to provide bright spots for graphic design. Throughout the development of history, due to the constant changes in the social environment, the expression of beauty is constantly changing, but at its root, the essence of beauty will not change. For the perceptual beauty in the color of oil painting, each individual has a huge "common sense of power". If it is excavated and expressed by the artist, it will have the power to transcend reality and directly stimulate the soul [3]. In recent years, there have been many artists who have emphasized that in the performance of oil paintings, medicines are constantly going to be complicated and simple, paying attention to the expression of beauty, paying attention to the influence of imagination, and constantly pursuing their own individual beauty. This shows that the perceptual beauty of oil painting has a very important effect on the expression of art. It is also an important supplement to the design effect applied to modern graphic design.

\section{Conclusion}

The art of painting has a great connection with graphic design, and its elements are mutually infiltrated between the two, so the visual effect of painting art has been vividly reflected in the design of modern graphic advertising. In addition, in the design of modern print advertising, attention should also be paid to the application and expression of painting elements to form a good visual effect.

\section{Acknowledgements}

Fund Project: Funded by the Yunnan Provincial Department of Education Science Research Fund (Project No. 2017ZZX259) 


\section{References}

[1] Liu Sheng, Zhu Zhongquan, Wang Yeping. Exploring the application of ink elements in domestic advertising design [J]. Packaging World, 2011 (01): 67-68+71.

[2] Yu Fei. Discussion on the Application of Traditional Ink Elements in Modern Graphic Design [J]. Business Culture, 2011(03):296.

[3] Huang Aimin. Application of traditional ink and wash elements in modern graphic design [J]. Literary Review, 2013(05): 115-117.

[4] Liu Yan, Zhu Ying. Research on the Application of Chinese Traditional Ink Painting Elements in Modern Advertising Design [J]. Popular Literature, 2015(18): 143.

[5] Hu Yajing. On the application and innovation of traditional ink elements in modern graphic design [J]. The House of Drama, 2016 (02): 150-151. 